\title{
A deterministic width function model
}

\author{
C. E. Puente and B. Sivakumar \\ Department of Land, Air \& Water Resources, University of California, Davis, USA
}

Received: 11 September 2002 - Revised: 13 March 2003 - Accepted: 15 May 2003

\begin{abstract}
.
Use of a deterministic fractal-multifractal (FM) geometric method to model width functions of natural river networks, as derived distributions of simple multifractal measures via fractal interpolating functions, is reported. It is first demonstrated that the FM procedure may be used to simulate natural width functions, preserving their most relevant features like their overall shape and texture and their observed power-law scaling on their power spectra. It is then shown, via two natural river networks (Racoon and Brushy creeks in the United States), that the FM approach may also be used to closely approximate existing width functions.
\end{abstract}

\section{Introduction}

The width function, $w(r)$, defined such that $w(r) d r$ is the area of a basin whose flow distance to the outlet is between $r$ and $r+d r$, is an important geomorphological qualifier of a river network. As such a function captures the essential features of the basin's response as implied by the instantaneous unit hydrograph (Rodriguez-Iturbe and Rinaldo, 1997; Gupta and Waymire, 1998), its proper modeling has become increasingly relevant.

Recently, a variety of sophisticated stochastic models for the width function have been introduced (Marani et al., 1994; Veneziano et al., 2000). While the work of Marani et al. (1994) relied on fractional Brownian motion and on random multiplicative cascades in order to capture, respectively, the power spectra and the multifractal characteristics of natural width functions; Veneziano et al. (2000) introduced an iterated random pulse (IRP) model that, while accounting explicitly for the self-similarity of natural river networks, properly captures their non-negativity, non-stationarity, and the power-law decay of their spectral density function.

The purpose of this article is to illustrate by means of few examples that a deterministic fractal-multifractal (FM) rep-

Correspondence to: C. E. Puente (cepuente@ucdavis.edu) resentation (Puente, 1992, 1994), successfully employed to model data sets corresponding to a host of geophysical processes, such as rainfall (Puente and Obregón, 1996; Obregón et al., 2002a, b), turbulence (Puente and Obregón, 1999), and groundwater contamination transport (Puente et al., 2001a, b), may also be used to represent the overall structure of the width function of natural catchments.

\section{The Fractal-Multifractal approach}

Figure 1 illustrates the construction of a derived distribution via the FM approach (Puente, 1992, 1994). As is seen, such a set named $d y$ is found transforming a (deterministic) binomial multifractal measure, $d x$, (bottom left), via a (deterministic) fractal interpolating function $f$. The set $d y$ is obtained considering all relevant events in $x$ corresponding to a level $y$ and adding their contributions, i.e. $d y(B)=d x\{x: f(x) \in B\}$, for all Borel subsets $B$ on $y$.

For a given set of $N+1$ points in the plane $\left\{\left(x_{0}, y_{0}\right),\left(x_{1}, y_{1}\right), \ldots,\left(x_{N}, y_{N}\right) ; x_{0}<x_{1}<\ldots<x_{N}\right\}$, measures $d x$ and $d y$ may be calculated iterating a suitable set of $N$ contractile affine mappings (according to an appropriate set of $N$ weights $p_{n}$ that dictate the intermittencies of $d x$ ) and counting over a desired number of bins (the resolution of the records to be fitted) over $x$ and $y$ (Puente, 1994). Such mappings have the specific form

$w_{n}\left(\begin{array}{l}x \\ y\end{array}\right)=\left(\begin{array}{cc}a_{n} & 0 \\ c_{n} & d_{n}\end{array}\right)\left(\begin{array}{l}x \\ y\end{array}\right)+\left(\begin{array}{l}e_{n} \\ f_{n}\end{array}\right)$,

where $a_{n}, c_{n}, e_{n}$, and $f_{n}$ are related to the interpolating points and the free scaling parameters $d_{n}, 0 \leq\left|d_{n}\right|<1$, via the conditions

$w_{n}\left(\begin{array}{l}x_{0} \\ y_{0}\end{array}\right)=\left(\begin{array}{l}x_{n-1} \\ y_{n-1}\end{array}\right)$

and

$w_{n}\left(\begin{array}{l}x_{N} \\ y_{N}\end{array}\right)=\left(\begin{array}{l}x_{n} \\ y_{n}\end{array}\right)$ 

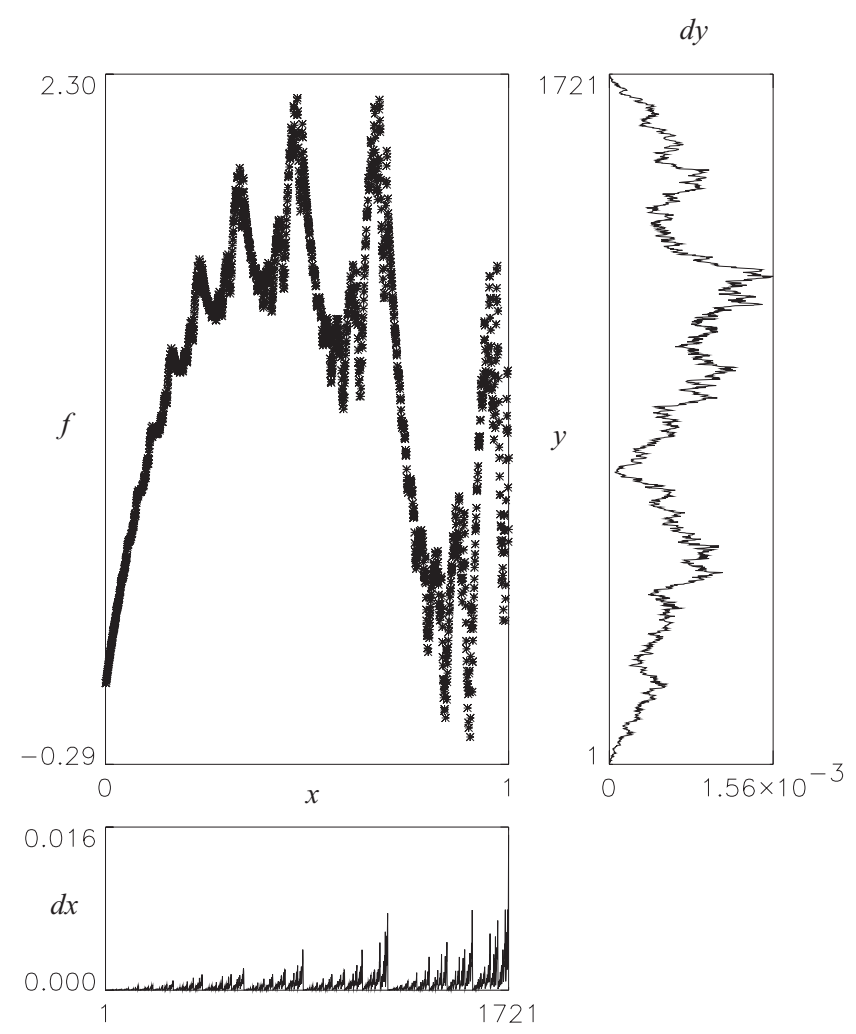

Fig. 1. The fractal-multifractal framework in two dimensions. A multifractal measure $d x$ is transformed via a fractal interpolating function $f$ into a derived measure $d y$.

for $n=1,2, \ldots, N$, and yield a fractal interpolating function whose graph's dimension $D f$ is: (a) 1 , if $\sum\left|d_{n}\right| \leq 1$, and (b) $\geq 1$, from $\sum\left|d_{n}\right| a_{n}^{D f-1}=1$, if $\sum|d n|>1$ (Barnsley, 1988).

A vast class of deterministic derived measures $d y$ may be obtained by varying the parameters of $f$ and $d x$, i.e. $\left(x_{n}, y_{n}\right), d_{n}$ and $p_{n}$, and by allowing projections to be found at directions other than $y$, i.e. at angles $\theta$ different from 0 degrees. At the end, the FM methodology results in a large universe of "data sets", that, depending on the fractal dimension $D f$, include a wide variety of multifractal records, even having "chaotic" or "stochastic" properties, and Gaussian distributions, in the limit when $D f$ tends to two (Puente, 2004). The geometric approach may easily be tailored to generate sets of arbitrary resolutions (e.g. Obregón et al., 2002b) which turn out to have the desirable feature of being neither self-similar nor self-affine (as seen in Fig. 1), as found in many practical applications.

It so happens that the set $d y$ in Fig. 1 (and many others) has a similar shape and texture as found in width functions of natural river networks and such observation motivates using the FM approach to represent natural width functions. In what follows, it is shown how the FM approach may indeed be employed to simulate general width functions and to model specific width functions as observed in nature.
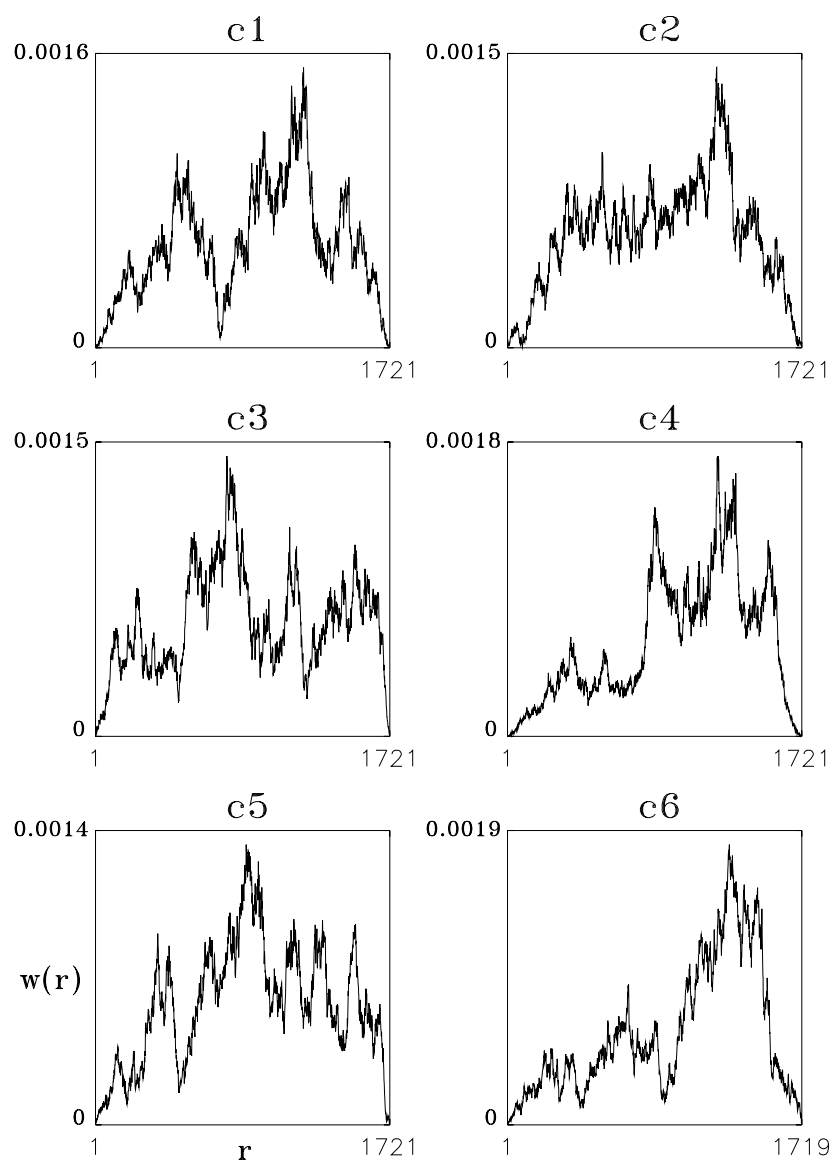

Fig. 2. Examples of (normalized) width functions, $c 1$ to $c 4$ are generated via the FM procedure, while c5 and c6 correspond to Racoon and Brushy creeks.

\section{Simulated and natural width functions}

As explained in Veneziano et al. (2000), natural width functions possess three important characteristics: (a) nonnegativity, (b) non-stationarity, and (c) power-law decay of the spectral density function, $S(f) \sim f^{-\beta}$, with values of $\beta$ typically ranging between 1.6 and 2.0. To illustrate such features, Fig. 2 includes six such plausible graphs (normalized so that their areas equal one unit), cases $\mathrm{c} 1$ to $\mathrm{c} 6$, made of 1721 data points for $\mathrm{c} 1$ to $\mathrm{c} 5$ and 1719 points for $\mathrm{c} 6$, and Fig. 3 shows their corresponding power spectra.

As seen in Fig. 2, all such graphs are indeed non-negative, they possess similar high-frequency textures, and they are also non-stationary as they begin and end at zero. As shown in Fig. 3, the power spectra of these sets have similar decay and their exponents $\beta$ are bounded by the value of 2.0 , as shown by the straight lines on all graphs.

It happens that while the first four sets in Fig. 2 were obtained via the aforementioned deterministic FM methodology and are given by fractal interpolating functions whose graphs have dimensions of, in order, 1.42, 1.48, 1.52 and 1.62 (see Table 1), the last two ( $\mathrm{c} 5$ and $\mathrm{c} 6$ ) correspond to two natural catchments: Racoon creek of $384 \mathrm{~km}^{2}$ located in 

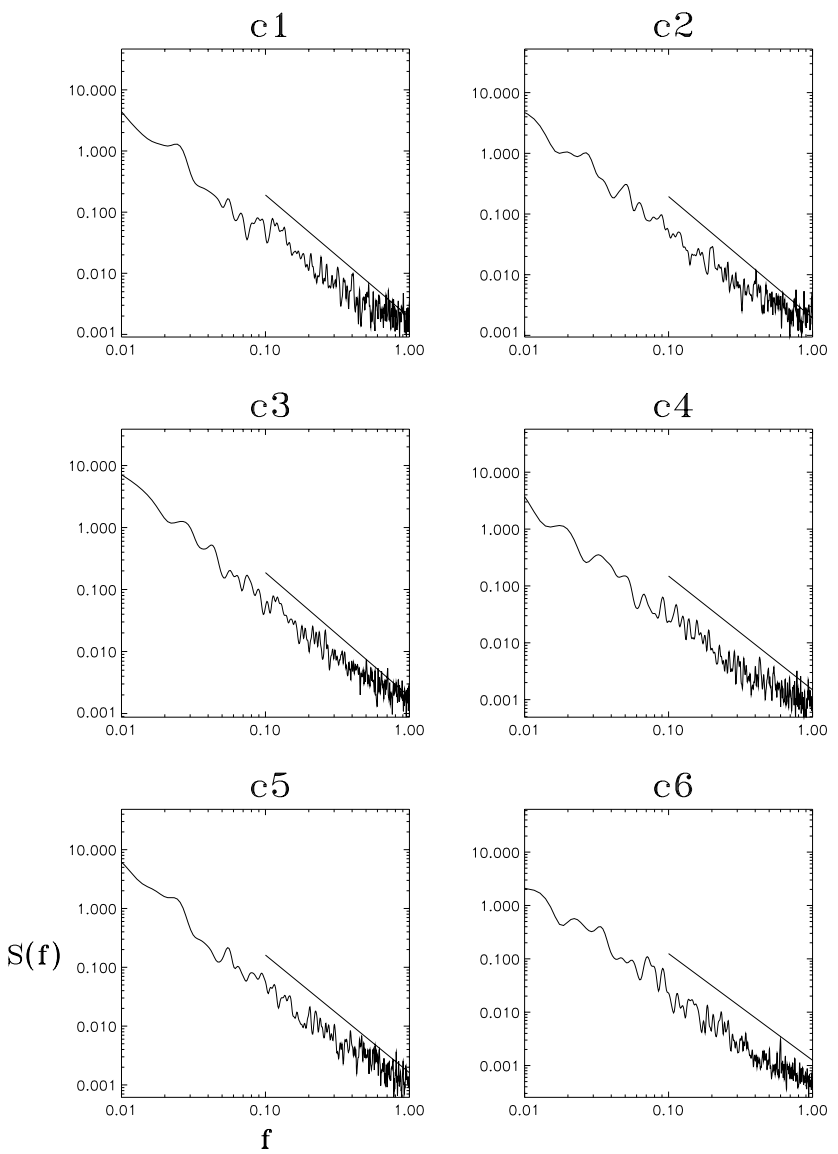

Fig. 3. Power spectra of the width functions shown in Fig. 2.

Table 1. FM parameters for simulated width functions $\mathrm{c} 1$ to $\mathrm{c} 4$ as shown in Fig. 2. Values not presented below are set as follows: $x_{0}=0, y_{0}=0$, and $x_{2}=1$.

\begin{tabular}{ccccccc}
\hline "Basin" & \multicolumn{5}{c}{ Coordinates } & Angle $\theta$ \\
\hline & $x_{1}$ & $x_{3}$ & $y_{1}$ & $y_{2}$ & $y_{3}$ & \\
\hline c1 & 0.700 & & 1.800 & 0.800 & & 0.0 \\
c2 & 0.700 & 1.500 & 1.800 & 0.800 & 0.000 & 8.0 \\
c3 & 0.600 & & 2.000 & 1.000 & & 0.0 \\
c4 & 0.600 & & 2.000 & 1.300 & & 12.0 \\
\hline
\end{tabular}

\begin{tabular}{|c|c|c|c|c|c|c|}
\hline \multirow[t]{2}{*}{ "Basin" } & \multicolumn{3}{|c|}{ Scalings } & \multicolumn{3}{|c|}{ Intermittencies } \\
\hline & $d_{1}$ & $d_{2}$ & $d_{3}$ & $p_{1}$ & $p_{2}$ & $p_{3}$ \\
\hline $\mathrm{c} 1$ & 0.600 & -0.800 & & 0.500 & 0.500 & \\
\hline c2 & 0.540 & -0.730 & -0.500 & 0.440 & 0.330 & 0.230 \\
\hline c3 & -0.800 & -0.630 & & 0.510 & 0.490 & \\
\hline $\mathrm{c} 4$ & -0.890 & -0.630 & & 0.470 & 0.530 & \\
\hline
\end{tabular}

Table 2. FM parameters for approximated width functions of Racoon and Brushy creeks as depicted in Fig. 4. Values not presented below are set as follows: $x_{0}=0, y_{0}=0$, and $x_{2}=1$

\begin{tabular}{|c|c|c|c|c|}
\hline \multirow[t]{2}{*}{ Basin } & \multicolumn{3}{|c|}{ Coordinates } & \multirow[t]{2}{*}{ Angle $\theta$} \\
\hline & $x_{1}$ & $y_{1}$ & $y_{2}$ & \\
\hline Racoon & 0.607 & 2.015 & 1.095 & -0.67 \\
\hline Brushy & 0.550 & 2.494 & 2.077 & 9.90 \\
\hline \multirow[t]{2}{*}{ Basin } & \multicolumn{2}{|c|}{ Scalings } & \multicolumn{2}{|c|}{ Intermittencies } \\
\hline & $d_{1}$ & $d_{2}$ & $p_{1}$ & $p_{2}$ \\
\hline Racoon & -0.881 & -0.615 & 0.530 & 0.470 \\
\hline Brushy & -0.917 & -0.653 & 0.492 & 0.508 \\
\hline
\end{tabular}

Pennsylvania and Brushy creek of $322 \mathrm{~km}^{2}$ situated in Alabama, both extending for about $50 \mathrm{~km}$ from their respective outlets (Veneziano et al., 2000). These results show that the FM approach provides, by varying its key parameters, great many outcomes that may be considered suitable representations (simulations) of natural width functions, simulations which, by varying smoothly with respect to their parameters, provide an opportunity to represent natural width functions, at any given resolution, in their entirety.

In what follows, it shall be shown how the FM approach may be used to find suitable approximations of natural width functions, considering the aforementioned creeks.

\section{FM approximations to Racoon and Brushy creeks}

This section presents the results obtained while solving the inherent inverse problem to approximate the width functions for the Racoon and Brushy creeks.

Based on previous experience with other data sets (Obregón et al., 2002a, b), an objective function accounting for the cumulative distributions of the records and their derivatives was minimized, performing the search via a genetic algorithm (Duan et al., 1992). Specifically, an objective function $\Delta$ was used adding: (a) the sum of square differences between cumulative distributions of real (normalized) width functions and FM sets $\left(\sum \Delta^{2}\right)$; and (b) the sum of square differences between cumulative distributions of normalized absolute one-lag derivatives of real (normalized) width functions and FM sets $\left(\sum \Delta_{d}^{2}\right)$.

While Table 2 includes the obtained parameters from the optimization exercise for both catchments, Fig. 4 summarizes pictorially their results, as it contains: (a) the normalized width functions (so that their areas are equal to one unit); (b) their cumulative distributions; and (c) their power spectra. 

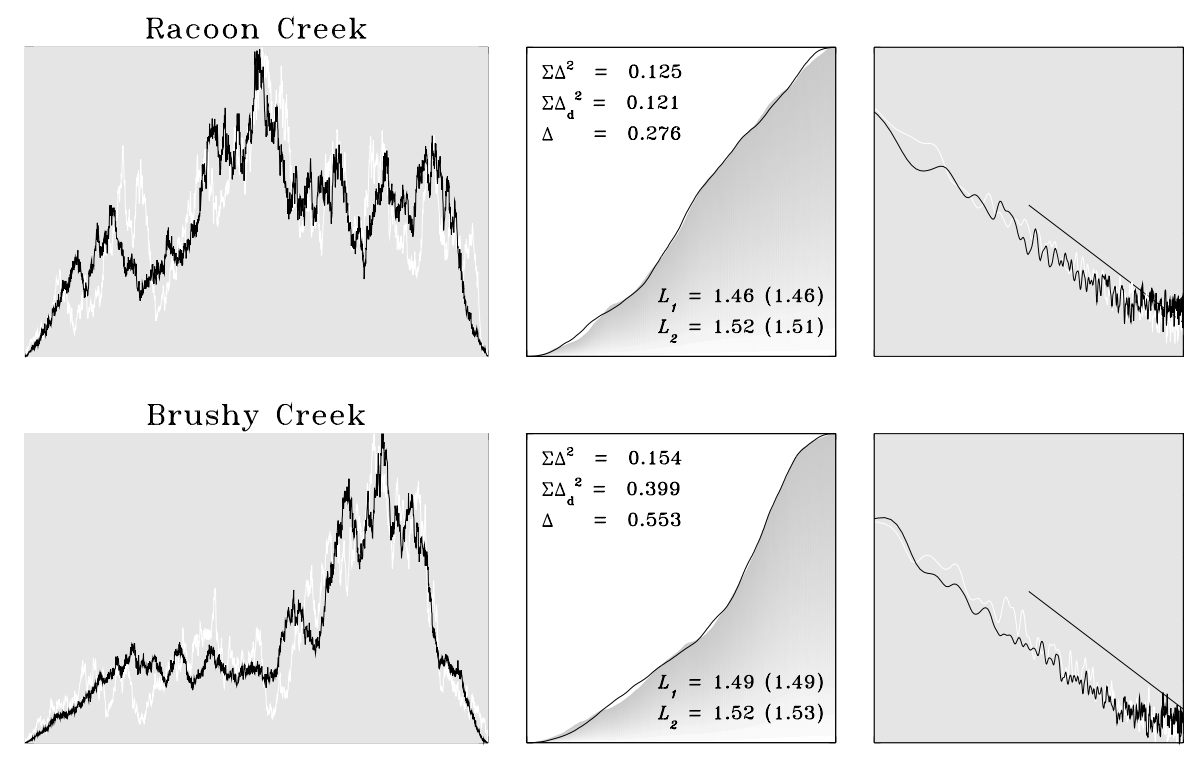

Fig. 4. Actual and FM fitted width functions, cumulative distributions, and power spectra for Racoon and Brushy creeks. For the actual sets, the width function and the power spectra are shown in white, whereas the cumulative distribution is highlighted in gray. The horizontal and vertical scales for the three graphs corresponding to Racoon creek are: [1, 1721], [0, 0.0014]; [0, 1], $[0,1]$; and $[0.01,1.0],[0.001,10.0]$ as in $\mathrm{c} 5$ in Fig. 3. Those scales for Brushy creek are: [1, 1719], [0, 0.0019]; [0,1], $[0,1]$; and $[0.01,1.0],[0.001,10.0]$, as in case $\mathrm{c} 6$ in Fig. 3.
As may be inferred, the FM representations for the Racoon and Brushy creek catchments are based on binomial multifractal measures and on fractal interpolating functions passing by three points (leading to seven free parameters, see Table 2, for $x_{0}, y_{0}$ and $x_{2}$ were kept fixed), whose graphs have dimensions of 1.60 and 1.66 , respectively. These results stem from very faithful descriptions of the records' cumulative distributions and of their derivatives (not shown), as implied by very low values of the attributes $\sum \Delta^{2}, \sum \Delta_{d}^{2}$, and hence $\Delta$ (all less than 1$)$, that started, as the search began, at values greater than 20 . The closeness of such fits may also be appreciated in the close agreement in the quantities $L_{1}$ and $L_{2}$ (real values in parenthesis), that give the lengths of cumulative distributions of the records and of their derivatives, when calculated from $(0,0)$ to $(1,1)$, such that the records' domain is set to one unit.

As may be seen in Fig. 4, the obtained FM width functions capture the overall trends present in the actual functions, including good approximations of the peaks, and possess similar textures that translate into good agreement of the power-law decay in the power spectra for both catchments. Notice that this happens despite small deviations from the scaling lines at high frequencies, as the FM approximations shown, that do not optimize the desired scaling law, turned out to produce smoother width functions compared to the actual sets.

As typical width functions share similar features to the ones included here (see for example Rodriguez-Iturbe and Rinaldo, 1997), the results in Fig. 4 further illustrate that the FM approach may be used to model them, using fractal interpolating functions whose graphs have fractal dimensions ranging between 1.4 and 1.7. As specific fits of other width functions may certainly be obtained in a similar fashion, it is envisioned that a "physical" meaning may be assigned to their FM geometric parameters (or groups of them) in terms of actual characteristics of the underlying watersheds, as it has been found in other applications (e.g. Puente et al., 2001a, b). Such an exercise may ultimately lead to a regional parameterization of a host of width functions.

\section{Conclusions}

This article has shown that the FM methodology may be employed to obtain suitable approximations of width functions of natural catchments as derived distributions of simple multifractal measures via fractal interpolating functions, in a rather parsimonious way. As such, it has been shown that such a deterministic procedure may be used to not only simulate sets that properly preserve all essential features, as captured by stochastic models (Veneziano et al., 2000), but also fit specific width function sets, which random models, due to their specific random structure, cannot easily do.

The FM approach, while providing parsimonious descriptions of whole width functions leading to vast compression ratios (1721 to 7 or about 246 to 1 in the examples considered), represents a new approach for understanding the complexities of natural width functions such that their inherent geometries are fully captured and at any given resolution. Due to its scope, the FM method cannot be safely compared with other existing (stochastic) procedures that by definition are able to simulate plausible realizations of width functions (even if with fewer parameters than a wholistic FM representation may have). In a sense, the FM methodology, by representing specific width functions, just parameterizes realizations as given by stochastic representations.

Given that space-filling Peano river basins yield simple multifractal width functions that correspond to the parent measure of the FM procedure (Marani et al., 1991), it is envisioned that further analysis of other width functions using the FM approach may provide, via the parameters of the inherent fractal interpolating functions, a plausible physical interpre- 
tation of the geometric parameters and a subsequent regional classification of river networks.

Acknowledgements. The work presented in this article was supported in part by the USEPA via Grant GAD \# R82 4780 and by NASA under Grant NAG5-7441.The data sets used in this work were kindly provided to us by Glenn E. Moglen. Comments by anonymous reviewers are gratefully acknowledged.

\section{References}

Barnsley, M. F.: Fractals Everywhere, Academic, San Diego, Calif., 1988.

Duan, Q., Sorooshian, S., and Gupta, V.: Effective and efficient global optimization for conceptual rainfall-runoff models, Water Resour. Res., 28(4), 1015-1031, 1992.

Gupta, V. K. and Waymire, E. C.: Spatial variability and scale invariance in hydrologic regionalization, in Scale Dependence and Invariance in Hydrology, edited by G. Sposito, Cambridge Univ. Press, New York, 88-135, 1998.

Marani, A., Rigon, R., and Rinaldo, A.: A note on fractal channel networks, Water Resour. Res., 27, 3041-3049, 1991.

Marani, M., Rinaldo, A., Rigon, R., and Rodríguez-Iturbe, I.: Geomorphological width functions and the random cascade, Geophys. Res. Lett., 21(19), 2123-2126, 1994.

Obregón, N., Sivakumar, B., and Puente, C. E.: A deterministic geometric representation of temporal rainfall: Sensitivity analysis for a storm in Boston, J. Hydrol., 269(3-4), 224-235, $2002 \mathrm{a}$.

Obregón, N., Puente, C. E., and Sivakumar, B.: Modeling high resolution rain rates via a deterministic fractal-multifractal approach, Fractals, 10(3), 387-394, 2002b.

Puente, C. E.: Multinomial multifractals, fractal interpolators, and the Gaussian distribution, Phys. Lett. A, 161, 441-447, 1992.

Puente, C. E.: Deterministic fractal geometry and probability, Int. J. Bifurcations Chaos, 4(6), 1613-1629, 1994.

Puente, C. E.: A universe of projections: May Plato be right?, Chaos, Solitons and Fractals, 19/2, 241-253, 2004.

Puente, C. E. and Obregón, N.: A deterministic geometric representation of temporal rainfall: Results for a storm in Boston, Water Resour. Res., 32(9), 2825-2839, 1996.

Puente, C. E. and Obregón, N.: A geometric platonic approach to multifractality and turbulence, Fractals, 7(4), 403-420, 1999.

Puente, C. E., Robayo, O., Díaz, M. C., and Sivakumar, B.: A fractal-multifractal approach to groundwater contamination, 1. Modeling conservative tracers at the Borden site, Stoch. Env. Res. Risk Assess., 15(5), 357-371, 2001a.

Puente, C. E., Robayo, O., and Sivakumar, B.: A fractal-multifractal approach to groundwater contamination, 2. Predicting conservative tracers at the Borden site, Stoch. Env. Res. Risk Assess., 15(5), 372-383, 2001b.

Rodríguez-Iturbe, I. and Rinaldo, A.: Fractal River Basins: Chance and Self-organization, Cambridge Univ. Press, New York, 1997.

Veneziano, D., Moglen, G. E., Furcolo, P., and Iacobellis, V.: Stochastic model of the width function, Water Resour. Res., 36(4), 1143-1157, 2000. 